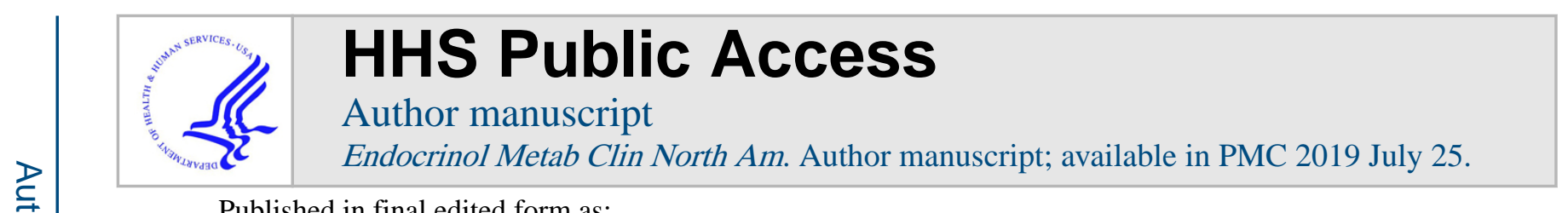

Published in final edited form as:

Endocrinol Metab Clin North Am. 2013 December ; 42(4): 809-832. doi:10.1016/j.ecl.2013.07.006.

\title{
Gastrointestinal Complications of Diabetes
}

\author{
Brigid S. Boland, MD ${ }^{a}{ }^{,}$, Steven V. Edelman, MD $^{b, c}$, James D. Wolosin, MD ${ }^{d}$ \\ aDivision of Gastroenterology, Department of Medicine, University of California, San Diego, 9500 \\ Gilman Drive, La Jolla, CA 92093, USA; \\ bivision of Endocrinology and Metabolism, University of California, San Diego, 9350 Campus \\ Point Drive, Suite 2G, La Jolla, CA 92093-9111G, USA; \\ 'Division of Endocrinology and Metabolism, Diabetes Care Clinic, Veterans Affairs Medical \\ Center, 3350 La Jolla Village Drive (111G), San Diego, CA 92161, USA; \\ dDivision of Gastroenterology, Sharp Rees-Stealy Medical Group, 2929 Health Center Drive, San \\ Diego, CA 92123, USA
}

\section{Keywords}

Gastrointestinal symptoms; Glycemic control; Gastroparesis; Celiac; Nonalcoholic fatty liver disease

\section{INTRODUCTION}

When practitioners think about complications of diabetes, they may focus on the microvascular, macrovascular, and peripheral neuropathic complications that are known to be associated with the disease. Although gastrointestinal problems are extremely common, with upper gastrointestinal symptoms alone affecting more than $40 \%$ of the general population, ${ }^{1}$ the incidence of certain gastrointestinal symptoms is more common in diabetes, and health care professionals should be aware of these associations. Gastrointestinal symptoms have not only a detrimental effect on quality of life but also significant medical consequences. Poor control of diabetes can affect any segment of the gut from the mouth to the rectum. However, unfamiliarity with these symptoms can delay treatment or referrals to appropriate specialists. The purpose of this review is to highlight the incidence, diagnosis, and treatment of some of the more common gastrointestinal complications of diabetes.

\section{ESOPHAGEAL DISORDERS}

\section{Esophageal Motility Dysfunction in Diabetes}

The term esophageal motility dysfunction is a phrase used to describe a multitude of esophageal complications that can occur in diabetic patients. Abnormalities seen include reduced lower esophageal sphincter tone, diminished amplitude of esophageal contractions,

*Corresponding author.: bboland@ucsd.edu.

Conflict of Interest: None. 
reduced coordination of esophageal contractions, prolonged esophageal transit, and increased acid reflux. ${ }^{2}$ Esophageal motility disorders appear to be driven by neuropathy, a known complication of diabetes. Specifically, vagal nerve dysfunction is thought to drive the underlying pathophysiology. However, recent studies have also implicated motor nerve dysfunction as playing a role. ${ }^{3}$

\section{Symptoms of Esophageal Motility Disorders}

Symptoms of esophageal motility disorders may include heartburn after eating and/or drinking, chest pain, odynophagia, and dysphagia. More commonly, these abnormalities are asymptomatic but have been incidentally observed in clinical studies. Treatment options for esophageal hypomotility are limited but may entail lifestyle modification.

\section{Gastroesophageal Reflux Disease in Patients with Diabetes}

Just as many motility abnormalities are clinically silent in diabetic patients, the prevalence of asymptomatic gastroesophageal reflux confirmed by $\mathrm{pH}$ study is significantly higher in diabetic patients than in healthy controls. ${ }^{4}$ Furthermore, the prevalence of gastroesophageal reflux disease (GERD) is higher in patients with diabetes with neuropathy as compared with the general population (41\% as compared to $14 \%) .{ }^{5}$ Practitioners should be mindful of this association and be aware of the incidence of GERD when a diagnosis of neuropathy is established. As GERD is a common clinical entity, it is worth familiarizing one's self with the common presentation and treatment modalities.

\section{Management of GERD}

In patients with reflux, initial recommendations should include lifestyle modifications, such as avoidance of trigger foods (coffee, tomato sauce, spicy foods, and alcohol being common offenders), reduction in meal size, avoidance of eating before sleeping, smoking cessation, elevation of head at night, and weight loss. Frequently, patients will require medical therapy. Management may entail antacids as needed or $\mathrm{H} 2$ blockers, such as rantidine, for mild disease. For more significant or persistent symptoms, proton pump inhibitors (PPIs), such as omeprazole or pantoprazole, are extremely effective in the treatment of reflux. Interestingly, a recent randomized clinical trial showed that PPIs improve glycemic control, suggesting additional benefits of PPIs in diabetic patients with GERD. ${ }^{6}$ In patients with classic symptoms of postprandial reflux that responds to antacids, further diagnostic testing is not necessary.

\section{Diagnostic Testing for GERD}

When the symptoms of GERD do not respond to a PPI, the underlying diagnosis should be questioned. A referral to a gastroenterologist is appropriate to guide further diagnostic evaluation, such as upper endoscopy, 24-hour esophageal $\mathrm{pH}$ monitoring, or manometry. Esophageal $\mathrm{pH}$ monitoring directly measures acid in the esophagus and quantifies acid reflux, providing a definitive diagnosis. Technological advances have significantly enhanced diagnostic capabilities with the development of a wireless $\mathrm{pH}$-detection capsule that is placed during endoscopy. Manometry, a study that measures esophageal pressure and muscular contractions, may be used to evaluate for a motility disorder causing GERD-like 
symptoms. In patients with a long-standing history of GERD, especially middle-aged, overweight Caucasian men, upper endoscopy should be considered to evaluate for Barrett esophagus and assess for esophageal cancer risks.

\section{Esophageal Candidiasis}

Individuals with diabetes are at higher risk for oral or esophageal candidiasis that is typically caused by Candida albicans. Poor glycemic control allows for favorable conditions for the yeast to grow, increasing the risk of fungal infection. The incidence of esophageal candidiasis in patients with diabetes is not well defined.

\section{Management of Oropharyngeal and Esophageal Candidiasis}

Candidiasis limited to the oropharyngeal cavity may be treated with nystatin. A typical dosage is 4 to $6 \mathrm{~mL}$ (400,000 to 600,000 units) 4 times per day in a swish-and-swallow fashion. Patients continue this therapy until 48 hours after symptoms have resolved. However, if the symptoms are not responding, oral fluconazole should be the treatment of choice. Similarly, the presence of odynophagia in combination with oral thrush is highly predictive of esophageal involvement and is an indication for fluconazole therapy. Therefore, the presence of thrush should always key a practitioner to inquire about pain with swallowing. A loading dose of fluconazole $200 \mathrm{mg}$ is given, followed by 100 to $200 \mathrm{mg}$ daily for 7 to 14 days. Symptoms should resolve in 1 week with treatment, and lack of response should prompt further evaluation with upper endoscopy. ${ }^{7}$

\section{GASTRIC DISORDERS}

\section{Gastroparesis}

Gastroparesis is defined as delayed gastric emptying in the absence of mechanical obstruction. The condition can result in a wide array of symptoms, ranging from none to a complete inability to tolerate oral nutrients with chronic nausea and vomiting. Delayed gastric emptying may be seen in up to $40 \%$ of patients with diabetes but only $10 \%$ or fewer of these patients will have any symptoms. Severe symptoms can result in a significant decrease in quality of life and can further potentiate poor glycemic control, as matching mealtime insulin with slow emptying can be extremely difficult. Patients will often complain of early satiety that may be accompanied by postprandial nausea, vomiting, belching, reflux, palpitations, and/or abdominal pain. The tremendous overlap with symptoms of functional dyspepsia may obscure the diagnosis of gastroparesis.

Although gastroparesis does not independently increase mortality, it is associated with a worse prognosis as compared with age-matched and gender-matched individuals with normal gastric emptying. The 5-year survival of individuals with gastroparesis is $67 \%$ as compared with the expected $81 \%$ survival. $^{8}$

\section{Epidemiology}

The overall incidence of gastroparesis is $4.8 \%$ in type 1 diabetes mellitus (DM1), $1 \%$ in type 2 diabetes mellitus (DM2), and $0.1 \%$ in the general population.9 Although the incidence of gastroparesis is higher in patients with DM1 as compared with DM2, the overall higher 
prevalence of DM2 makes gastroparesis more commonly seen by health care providers in this patient population. The incidence and prevalence in women is nearly 4 times that in men. ${ }^{8}$ Although onset of gastroparesis appears to occur after 10 years of disease duration, a recent population-based study found no association between diabetes duration and gastroparesis. ${ }^{9-11}$

\section{Pathophysiology of Gastroparesis}

Normal gastric emptying is a complex process involving synchronization of smooth muscle and autonomic nerves. This process is coordinated in the interstitial cells of Cajal (ICC), or the pacemakers of the stomach. The ICCs integrate fundic tone, antral contractions, and relaxation of the pylorus to facilitate postprandial emptying. Consequences of diabetes may lead to disruptions in different components of this process, interfering with the normal function of the stomach. Hyperglycemia, even at modest levels, disrupts gastric coordination and emptying in healthy volunteers as well as diabetic patients. ${ }^{12}$ Reductions in ICC quantity are seen in biopsies from individuals with gastroparesis. ${ }^{13}$

Autonomic neuropathy, inflammation, and oxidative stress appear to be critical components driving patients with diabetic gastroparesis. ${ }^{11}$ The effects of autonomic neuropathy have been demonstrated by gastric biopsies from diabetic patients with gastroparesis showing reduced number of nerve fibers. ${ }^{14}$ Vagal nerve functional studies are abnormal in diabetic patients with gastroparesis, showing reduction in postprandial gastric acid section. ${ }^{15}$ Oxidative stress has been implicated, given that loss of nitric oxide from enteric cells has been documented in mouse and human models of patients with diabetic gastroparesis and appears to occur early after diabetes onset. ${ }^{16,17}$ Similarly, loss of a cytoprotective enzyme, heme oxidase-1, has been linked to the pathogenesis of gastroparesis in mouse models. ${ }^{18}$ Further, oxidative stress and loss of survival signals from insulin and insulinlike growth factor (IGF)-1 contribute to ICC death. ${ }^{11,18}$

Overall, the symptoms lack sensitivity and specificity, but nausea, vomiting, and early satiety are the best predictors of gastroparesis. ${ }^{19}$ The presence of food in the stomach on endoscopy after a 12-hour fast suggests delayed gastric emptying and should raise suspicion for gastroparesis. Consensus guidelines recommend diagnostic testing to confirm the diagnosis of gastroparesis, and scintigraphy is typically the test of choice. ${ }^{20}$ For this examination, patients ingest radioisotope technetium- 99m-labeled test meals of defined caloric amount. After ingestion of the solid- phase meal, images will be obtained at 60 minutes, 120 minutes, 180 minutes, and 240 minutes. The most reliable predictor of gastroparesis is percentage of retained food at 4 hours. If possible, medications that delay or accelerate gastric emptying, should be discontinued approximately 48 to 72 hours before the study. Acute hyperglycemia may delay gastric emptying, so practitioners may want to delay scintigraphy until blood sugar has been stabilized. ${ }^{21}$ Many patients with diabetes may have abnormal emptying studies in the absence of symptoms, and others may have mildly abnormal gastric emptying along with functional dyspepsia that is not directly related to diabetes. It is important to take a good history and be certain that symptoms are suggestive of delayed gastric emptying before making a diagnosis of gastroparesis. 


\section{Nutritional Optimization}

Gastroparesis may interfere with oral intake and nutritional status; therefore, the first goals of management are to restore hydration, replete electrolytes, and ensure sufficient long-term oral intake. Alcohol and cigarette smoking should be avoided, as they slow gastric emptying. Dietary modifications for gastroparesis aim to optimize emptying of the stomach, while avoiding foods that may delay motility, such as those with high fat and fiber content. A gastroparesis diet entails small, frequent, low-fat, and low-residue meals. If individuals are unable to tolerate modified diets, liquid meals may be trialed, as gastric emptying of liquids is frequently maintained in gastroparesis. Consultation with a nutritionist may be quite useful in helping patients deal with multiple dietary restrictions. In severe cases, unintentional weight loss greater than $10 \%$ of body weight over 3 to 6 months suggests refractory gastroparesis and should prompt consideration for feeding tube placement. To bypass abnormal gastric motility, feeding tubes should be placed beyond the pylorus to bypass the abnormal motility in the stomach. ${ }^{20}$

\section{Management of Glycemic Control in Gastroparesis}

Based on experimental models, hyperglycemia delays gastric emptying in patients with diabetes as well as healthy patients. ${ }^{12,22}$ Management of gastroparesis should include optimization of glycemic control that may improve short-term symptoms. For patients on mealtime insulin, the timing of their mealtime bolus may have to be adjusted. Rapid-acting insulin can be dosed at the start of the meal or 15 minutes after starting a meal in an attempt to match peak insulin levels with glucose appearance. Similarly, switching from rapid-acting analogues to regular insulin may be appropriate for the same reason. Using the square and dual-wave bolus function that is a feature with insulin pumps can be helpful. In addition, current medical treatments, including pramlintide and GLP-1 analogs, cause delayed gastric emptying and may exacerbate symptoms of gastroparesis. ${ }^{23,24}$ The drugs may still be used in mild cases, but their use should be discouraged in any case where prokinetic agents must be initiated.

\section{Management of Gastroparesis in Diabetes}

Metoclopramide is the first-line prokinetic drug for treatment of gastroparesis. Metoclopramide is a D2 dopamine receptor antagonist (with less potent stimulation of 5HT4) that increases lower esophageal sphincter tone, gastric pressure, and antral contractions that aids in emptying of the stomach. Metoclopramide also provides antiemetic action by acting centrally and inhibiting the D2 dopamine and 5-HT3 receptors in the chemoreceptor trigger zone, a center in the medulla that integrates sensory input and stimulates vomiting. ${ }^{25}$ Although this is the only prokinetic medication for gastroparesis that is approved by the Food and Drug Administration (FDA), its use is approved for only 3 months. The strongest evidence supporting use of metoclopramide comes from 4 placebocontrolled studies that showed improvement in symptoms ranging from $25 \%$ to $50 \%$. However, these studies were of shorter duration, on the order of 3 weeks. ${ }^{26-29}$ Prolonged use of the medication necessitates an evaluation of the benefits versus potential risks of ongoing therapy. 
Metoclopramide carries a black box warning for an increased risk of tardive dyskinesia; a disorder characterized by involuntary movements of the face or extremities induced by dopamine inhibition. ${ }^{25}$ Although the incidence of tardive dyskinesia is estimated at approximately $0.2 \%$, the potential irreversible nature of the condition makes even a low incidence concerning. Groups at higher risk for developing tardive dyskinesia include patients on higher doses, those of younger age, and women. ${ }^{30}$ Other side effects include QT prolongation, parkinsonian movements, akathisia, and hyper-prolactinemia. Guidelines on use of metoclopramide recommend use of a minimal effective dose beginning with $5 \mathrm{mg}$ before meals, monitoring for early signs of tardive dyskinesia, and drug holidays. ${ }^{31}$

Domperidone, a type II dopamine antagonist, has a similar mechanism of action as metoclopramide but with reduced central nervous system effects, and, thus, tardive dyskinesia. Three large randomized controlled trials with domperidone in patients with diabetic gastroparesis have been published and showed symptomatic improvement as compared with baseline or placebo. ${ }^{32-34}$ Dosing typically starts at $10 \mathrm{mg}$ with meals. Baseline electrocardiogram is recommended before initiation of therapy based on potential QT prolongation. Less common side effects also include prolactinemia, and potential drug interactions. Although the makers of domperidone have never applied for FDA approval, there are programs for obtaining this medication in the United States. ${ }^{20}$

Intravenous erythromycin lactobionate, a motilin agonist, has been shown to be effective in short-term treatment of hospitalized patients with diabetic gastroparesis. However, ongoing administration of intravenous or oral erythromycin over longer periods of time leads to tachyphylaxis by downregulation of receptors for motilin. ${ }^{35}$ In addition, erythromycin itself may induce abnormal gastric motility with resultant nausea and vomiting.

Another technique that has been used in clinical practice is endoscopic intrapyloric injection of botulinum toxin. Manometry studies show that the pylorus has increased tone in patients with gastroparesis. Injection of botulinum toxin to block neurotransmission showed promise in open-label trials, but a randomized clinical trial including diabetic patients showed improvement in gastric emptying without symptomatic improvement. ${ }^{36}$ Thus, routine use of botulinum toxin for the treatment of gastroparesis is not advised (Table 1). ${ }^{20}$

Management of the nausea and vomiting associated with gastroparesis typically relies on off-label use of medications. Therapies that are used include prochlorperazine, promethazine, ondansetron, scopolamine, and dronabinol; however, there are no evidencebased guidelines for management.

Pain remains extremely challenging, as opiates further slow gastric emptying and exacerbate symptoms. Clinical trials are lacking. Practitioners should discontinue opiates and recommend nonopiate alternatives, including tricyclic antidepressants (TCAs), tramadol, selective serotonin receptor inhibitors, or gabapentin. Low-dose tricyclic antidepressants and selective serotonin receptor inhibitors may modulate pain and improve glycemic control. TCAs have the added benefit of treating pain from coexisting peripheral neuropathy; however, this class of medications has varying anticholinergic effects that may worsen gastroparesis. $^{20}$ 


\section{Gastric Electric Stimulation for Refractory Gastroparesis}

The gastric electrical stimulator (GES) is a neurostimulator that is implanted surgically into the abdomen and provides high-frequency stimulation to the stomach. GES was approved by the FDA under the humanitarian device exemption. A randomized crossover trial showed a reduction in weekly vomiting frequency from 13 to 7 , but in the subgroup of patients with diabetes there were no significant differences in symptoms and vomiting frequency. ${ }^{37} \mathrm{~A}$ recent meta-analysis included 10 studies and concluded that GES improved symptoms with small mean differences in symptom scores, and diabetic gastroparesis was the most responsive to GES. ${ }^{38}$ Although randomized trials and availability are lacking, GES may be an option for refractory cases.

\section{Salvage and Alternative Therapies for Gastroparesis}

There is little evidence to guide management of refractory cases of gastroparesis; however, salvage therapies for symptom management may include venting gastrostomy with or without jejunostomy feeding tube or gastectomy. Alternative treatments remain under investigation with acupuncture showing promise based on symptom improvement in a randomized study. ${ }^{39}$

\section{Accelerated Gastric Emptying}

Accelerated gastric emptying has emerged as a new entity, but few studies have been done to further understand this condition. Vagal dysfunction impairs gastric accommodation, leading to elevated pressures and rapid emptying. Symptoms are typically consistent with dumping syndrome, with the developmental of abdominal discomfort, nausea, vomiting, and diarrhea within an hour of eating. Although patients may have disproportionately more difficulty with postprandial glucose control and weight loss, the symptoms may be difficult to distinguish from gastroparesis. Gastric transit measured by scintigraphy typically establishes the diagnosis. Treatments are not well defined at this time, but amylin analogs and glucagonlike peptide-1 (GLP-1) agonists that can slow gastric emptying may improve symptoms. ${ }^{11}$

\section{SMALL INTESTINAL DISORDERS}

\section{Celiac Disease Overview}

Celiac disease is an immune-mediated enteropathy in which ingestion of gluten leads to atrophy of the small intestinal villi and malabsorption. Celiac disease affects approximately $1 \%$ of the US population; however, this risk is much higher in patients with DM1. Approximately $3 \%$ to $8 \%$ of patients with DM1 have celiac disease. ${ }^{40-42}$ From 1951 to 2001, the incidence of celiac disease has increased incrementally; an increase that mirrors the increased incidence of DM1. ${ }^{43,44}$ In the vast majority of cases, the diagnosis of DM1 precedes that of celiac disease. ${ }^{45}$

\section{Pathogenesis of Celiac Disease}

Gluten is a storage protein derived from wheat, rye, or barley. Gluten has high proline and glutamine content, which makes digestion difficult; a-gliadin is among the poly-peptides that remain undigested in the small intestine. If there is a defect in the intestinal epithelium, then 
a-gliadin crosses into the lamina propria. In patients with celiac disease, a-gliadin initiates an innate and adaptive immune response that leads to an inflammatory infiltrate of the small bowel with villous destruction. The mechanism responsible for the epithelium defect is not well understood, but an infectious etiology has been proposed. ${ }^{46,47}$ In the lamina propria, tissue transglutaminase, a celiac auto-antigen, binds to gliadin and enhances its affinity for HLA DQ2 and DQ8 molecules on antigen-presenting cells that initiates humoral and cellmediated immune responses. The immune response leads to significant inflammation in the small intestine as well as distant organs. Simultaneously, activation of intraepithelial lymphocytes initiates an innate immune response contributing to the pathology. Ultimately, destruction of small intestinal enterocytes leads to villous atrophy and impaired nutritional absorption, causing the classic symptoms associated with celiac disease. ${ }^{46,47}$

\section{Genetic Predisposition to Celiac Disease}

Celiac disease essentially develops only in genetically predisposed individuals who possess certain HLA genes that encode for antigen-presenting cell surface markers. HLA DQ2 and DQ8 are the susceptibility serotypes that are present in $25 \%$ to $40 \%$ of the general population. Only a small subset of individuals with DQ2 or DQ8 will develop celiac disease. If an individual carries multiple susceptibility alleles, there is an additive risk of celiac disease. $^{48}$

\section{Association of Celiac Disease and DM1}

The mechanism underlying the increased incidence of celiac disease in patients with DM1 is not completely understood. Celiac disease and DM1 are autoimmune phenomena that share HLA and non-HLA susceptibility genes. A common environmental, microbial, or immunologic entity has been postulated, but has not been fully elucidated. Given this known association, diarrhea, weight loss, or new-onset hypoglycemic episodes in a patient with DM1 should prompt evaluation for celiac disease. The topic of whether to screen asymptomatic patients with DM1 for celiac disease remains somewhat controversial, although routinely occurs in the pediatric population. Furthermore, if an individual with DM1 undergoes upper endoscopy, obtaining duodenal biopsies is recommended to screen for celiac disease (Table 2). ${ }^{49}$

\section{Childhood Presentation of Celiac Disease}

Celiac disease may present with a wide variety of symptoms and historically was thought to be a disease of childhood. In infancy, typical presentations include development of abdominal pain and/or distension, steatorrhea, and vomiting after introduction of cereal into the diet. Falling off of the growth curve or failure to thrive should elicit concern for celiac disease. Older children may present with anemia or nutritional deficiencies, or in patients with diabetes, sudden improvement in hemoglobin A1c should prompt evaluation for celiac disease.

Celiac symptoms are predominantly related to malabsorption. The severity of diarrhea and weight loss correlates with the degree of the intestinal inflammation. However, symptoms of celiac disease may be nonspecific and frequently overlap with symptoms of irritable bowel 
syndrome. It is important to consider the diagnosis of celiac disease in any patient undergoing evaluation for irritable bowel syndrome.

\section{Extraintestinal Manifestations of Celiac Disease}

Adults with celiac disease may present with extraintestinal manifestations of the systemic disease, rather than classic gastrointestinal symptoms. Iron deficiency anemia can result from decreased iron absorption in the proximal small intestine. Less frequently, celiac disease will affect the terminal ileum and interfere with vitamin B12 absorption. Nutritional deficiencies may lead to other extraintestinal signs or symptoms, such as peripheral neuropathy, ataxia, and osteoporosis. The most common dermatologic manifestation of celiac disease is dermatitis herpetiformis, a pruritic, erythematous blistering lesion located on extensor surfaces. Gluten mediates the pathogenesis of dermatitis herpetiformis, and recent studies suggest that immunoglobulin A ( $\operatorname{Ig} \mathrm{A})$ antiepidermal tissue transglutaminase antibody deposition in the dermis may cause the rash. ${ }^{50}$

\section{Diagnosis of Celiac Disease: Utility of Serologic Testing}

The first step in evaluating for celiac disease should be serologic testing; however, definitive diagnosis of celiac disease is made by duodenal biopsies in the setting of gluten exposure. Current guidelines recommend testing for tissue transglutaminase IgA (TTG IGA) as the preferred serologic test for celiac disease. Based on multiple studies, the overall specificity is greater than $95 \%$, with sensitivity ranging from $89 \%$ to $96 \% .51,52$ The antiendomysial antibody (EMA) test has a similar profile but is more expensive. Antigliadin antibodies are of limited value because of decreased sensitivity and specificity. Patients with IgA deficiency will not, however, have detectable serum TTG IGA antibodies. It may be reasonable to routinely check $\operatorname{IgA}$ levels when evaluating individuals at high risk for celiac with the TTG IGA antibody. In individuals with low or absent IgA levels, deamidated gliadin peptide IgG antibody may be a useful screening tool. In the setting of DM1, the overlapping susceptibility genes render HLA-DQ2 and DQ8 testing less useful.

\section{Duodenal Biopsies: Gold Standard for Diagnosis}

If serologies are positive, upper endoscopy with duodenal bulb and distal duodenal biopsies should be obtained to confirm the diagnosis. Upper endoscopy should also be performed in the setting of negative serologies if there is high clinical suspicion. Endoscopic appearance of the duodenum may be abnormal with scalloping or flattening of folds. Duodenal biopsies may reveal a spectrum of changes including crypt hypertrophy, villous atrophy, and a lymphocytic inflammatory infiltrate. Dietary intake at time of endoscopy must be assessed and taken into account when interpreting the results of the endoscopy, as reductions in gluten consumption may lessen the intestinal inflammation and damage. Initiation of a 4week gluten challenge may enable diagnosis of celiac disease in patients adhering to a gluten-free diet. 52,53

\section{Treatment of Celiac Disease: Gluten-Free Diet}

Elimination of dietary wheat, rye, and barley is the current therapy for celiac disease. A gluten-free diet has been shown to improve symptoms, improve mortality, and possibly 
reduce the risk of cancer. ${ }^{54,55}$ Nutritional consultation and support groups may offer assistance in helping patients adhere to a gluten-free diet. In a patient with concomitant diabetes, competing dietary restrictions may further complicate management of both diseases, and adequate nutrition remains a significant concern in the patients. Patients should be assessed for nutritional deficiencies in folic acid, vitamin B12, iron, calcium, and fatsoluble vitamins, as well as osteoporosis. ${ }^{46}$

\section{Failure of a Gluten-Free Diet}

Inadvertent exposure to gluten is the most common reason for failure to respond to a glutenfree diet. Failure should prompt reevaluation of the diagnosis of celiac disease and assessment of adherence to diet. If this evaluation is unrevealing, practitioners should consider alternative etiologies for the symptoms. Diseases that are associated with celiac disease and may cause similar symptoms include pancreatic insufficiency, small intestinal bacterial overgrowth, microscopic colitis, lactose intolerance, and irritable bowel syndrome. ${ }^{56} \mathrm{In}$ addition, celiac disease is associated with increased risk of small bowel adenocarcinoma and enteropathy-associated T-cell lymphoma. ${ }^{46}$

\section{Small Bowel Bacterial Overgrowth Overview}

Small bowel bacterial overgrowth (SIBO) is characterized by alterations in the type and quantity of bacteria within the small intestine. Significant changes in the microbiota of the small intestine may cause gastrointestinal symptoms, such as bloating, abdominal pain, diarrhea, and gas, and may lead to nutritional deficiencies.

\section{Epidemiology}

The prevalence of SIBO in the general population is difficult to estimate, although studies of healthy controls suggest a prevalence of $6 \%$ that increases with aging. ${ }^{57}$ In one recent study, $43 \%$ of patients with diabetes with diarrhea had SIBO and improved with antibiotic therapy. 58

\section{Pathogenesis}

The physiologic mechanisms for controlling intestinal bacterial growth include secretion of acid in the stomach and normal gastrointestinal motility. Specifically, in the small bowel, the migrating motor complex (MMC) clears any residual intestinal contents every 90 to 120 minutes. However, gastroparesis or abnormal motility of the small intestine enables bacterial stasis and subsequent overgrowth. Furthermore, any structural abnormalities from surgeries, particularly blind loops, may also create reservoirs that enable overgrowth of bacteria. The excessive growth of organisms leads to generation of ammonia, inflammatory cytokines, short-chain fatty acids, bile acid deconjugation, and toxins. Ultimately, the bacterial byproducts interfere with normal absorption of fat and carbohydrates. ${ }^{59}$

\section{Nutritional Complications of Untreated SIBO}

If the bacterial overgrowth is severe and goes untreated, individuals may eventually develop nutritional deficiencies. Bacterial deconjugation of bile salts creates bile acids that may cause direct toxic damage to villi. This process impairs absorption and disrupts micelle 
formation. Disruption of fat absorption leads to deficiencies in vitamins A, D, E, and K. In addition, anaerobic intestinal bacteria metabolize vitamin $\mathrm{B} 12$ before absorption can occur. 60

\section{Symptoms of Bacterial Overgrowth}

The typical symptoms associated with SIBO are abdominal pain with bloating, gas, and diarrhea. Other frequently reported symptoms include flatulence, abdominal distension, and weakness. The symptoms are frequently vague and overlap significantly with those of irritable bowel syndrome. Practitioners should consider this diagnosis when diabetic patients develop diarrhea and abdominal bloating.

\section{Jejunal Aspirate: Gold Standard for Diagnosis of SIBO}

SIBO is generally defined as greater than 100,000 colony-forming units(CFU)/mL in the small bowel as compared with the normal value of less than 10,000 CFU/mL. Historically, the gold standard for diagnosis is quantification of bacteria obtained from proximal jejunal aspirate during upper endoscopy; however, costs associated with endoscopy, rigorous specimen handling procedures, and difficulty culturing bacteria limit the widespread use of the test. ${ }^{61}$

\section{Use of Breath Tests in Diagnosis of SIBO}

Breath tests for diagnosis of SIBO are used to evaluate the ability of intestinal bacteria to produce analytes, such as hydrogen or radiolabeled carbon dioxide. In healthy individuals, glucose is absorbed in the small intestines; however, the excess bacteria in SIBO metabolize glucose into carbon dioxide and hydrogen that can be detected by breath test. The specificity and sensitivity of each specific test varies and fails in the setting of abnormal motility. In comparison with small bowel aspirate, the glucose hydrogen test has a sensitivity and specificity of $62 \%$ and $83 \%$, respectively. ${ }^{62}$ Given the low sensitivity, there is some debate over the utility of testing as compared with empiric treatment.

\section{Treatment of Underlying Etiology}

Treatment and reversal of the underlying etiology of SIBO may be difficult in certain circumstances. In the setting of gastroparesis or slow small intestinal transit as a predisposing factor, one should aim to optimize treatment of the motility disorder. Goals should include improvement of glycemic control and potential use of a prokinetic agent, such as metoclopramide or domperidone, as mentioned previously.

\section{Nutritional Assessment}

Individuals should be assessed for weight loss, malnutrition, or electrolyte imbalances. Significant weight loss should prompt discussion about nutritional supplementation. One may consider evaluating for deficiencies in fat-soluble vitamins and vitamin B12.

\section{Antibiotic Treatment in SIBO}

Antibiotic treatment for SIBO typically provides significant symptomatic relief. Although many different antibiotics are used, few randomized clinical trials have rigorously compared 
different regimens and durations. The most extensively studied medication in treatment of SIBO is rifaximin, a minimally absorbed antibiotic with activity against gram-negative and gram-positive bacteria. Based on a review of existing trials, rifaximin improved symptoms in $33 \%$ to $92 \%$ with eradication of SIBO in more than $80 \% .{ }^{63}$ Rifaximin, 1200 to $1600 \mathrm{mg}$ daily for 7 to 10 days, is the first-line treatment; however, other antibiotics, such as doxycycline, augmentin, and flagyl, are less expensive and frequently used in clinical practice with success. Recurrence of SIBO is common and may respond to repeated courses of antibiotics. In refractory cases, patients may require continuous cycling of antibiotics to control symptoms.

\section{Diabetic Diarrhea}

Diarrhea is a frequent gastrointestinal symptom encountered in diabetic patients with a wide variety of potential etiologies. Diarrhea may be related to ingestion of artificial sweeteners, concomitant celiac disease, bacterial overgrowth, irritable bowel syndrome, or medication side effects. The biguanide derivative, metformin, frequently causes diarrhea. This effect may occur either early or late in use. The a-glucosidase inhibitors, such as acarbose, may also cause diarrhea in a significant proportion of patients. Diabetic diarrhea typically occurs in insulin-dependent patients who have had diabetes for at least 8 years. Many of these patients suffer from peripheral and autonomic neuropathy. ${ }^{64,65}$

\section{Epidemiology of Diabetic Diarrhea}

Significant variation in estimates of the prevalence of diabetic diarrhea may be related to referral basis in tertiary care settings. The prevalence of diabetic diarrhea is higher in patients with DM1 when compared with DM2, with rates of $5.0 \%$ and $0.4 \%$ respectively. Factors associated with diabetic diarrhea are disease duration, A1c levels, male sex, and autonomic neuropathy. ${ }^{64}$ The lack of specific diagnostic markers for diabetic diarrhea makes differentiation from irritable bowel syndrome very difficult and undoubtedly there is some degree of overlap.

\section{Pathogenesis of Diabetic Diarrhea}

The pathogenesis of diabetic diarrhea is not well understood but appears to be closely related to autonomic neuropathy. The MMC, organized small bowel contractions while fasting, may be abnormal in patients with diabetes, slowing motility. Sympathetic denervation is found frequently in patients with autonomic neuropathy. Interruption of sympathetic nerves and adrenergic stimulation of electrolyte and fluid absorption may be the etiology of diarrhea. ${ }^{66}$

Diabetic diarrhea is typically watery, voluminous, and explosive, with or without steatorrhea. Although the symptoms of diabetic diarrhea may relatively straightforward, patients may accept these symptoms and fail to disclose these symptoms.

\section{Diagnosis}

Diabetic diarrhea is a diagnosis of exclusion, and diagnostic evaluation includes obtaining a detailed history about diet and medication use for alternative etiologies. Specifically, diarrhea is a frequent side effect of metformin. Diagnostic evaluation may include stool studies for parasitic infection, serology for celiac disease, and consideration of flexible 
sigmoidoscopy or colonoscopy to evaluate for inflammatory bowel disease or microscopic colitis. If there is a significant history of incontinence, one may consider anorectal manometry to evaluate defecation. ${ }^{64}$

\section{Treatment}

Treatment may begin with optimization of glycemic control and removal of medications that may be exacerbating symptoms. Medications are almost uniformly needed to control symptoms. Initial treatment should be empiric with use of standard antidiarrheal agents, such as loperamide, diphenoxylate with atropine, and codeine sulfate, as well as fiber supplementation. Cholestyramine may be of benefit, especially if there is a component of bile salt malabsorption. A trial of fiber supplementation or probiotics may also be helpful. The a2-adrenergic agonist, clonidine, may provide adrenergic stimuli to facilitate fluid and electrolyte absorption in the intestines that appears to be disrupted in diabetic diarrhea. Small studies demonstrated that clonidine reduced stool volume in diabetic patients with profuse diarrhea. ${ }^{67}$ Clonidine should be started at $0.1 \mathrm{mg}$ twice daily but may be increased to $0.6 \mathrm{mg}$ twice daily. If the medication is discontinued, a slow taper is recommended to prevent rebound hypertension. Octreotide, a long-acting somatostatin, has also been used to treat diabetic diarrhea with symptomatic improvement in small case series; however, it may slow small bowel motility and increase risk of bacterial overgrowth. ${ }^{68}$ Additionally, octreotide is an inhibitor of glucagon and insulin, which places patients with diabetes at risk for both hyperglycemia and hypoglycemia.

\section{LARGE INTESTINE DISORDERS}

\section{Fecal Incontinence}

Fecal incontinence, the involuntary passage of fecal matter, appears to be a consequence of autonomic neuropathy. Incidence in the general population is estimated at $1 \%$, although increases with age. ${ }^{69}$ Symptoms of fecal incontinence frequently start with concomitant lowvolume diarrhea. Nocturnal symptoms are frequent, and a subset of patients may have steatorrhea. Fecal incontinence typically occurs in patients with long disease duration and management is frequently challenging. Most of these patients are older and may have alternative etiologies for fecal incontinence, such as prior anal sphincter injuries from childbirth or surgery.

\section{Pathophysiology of Fecal Incontinence}

Autonomic neuropathy in diabetes appears to be responsible for nerve damage that leads to fecal incontinence. Multiple mechanisms help maintain fecal continence, and numerous insults are required to disrupt the process. Diabetic patients with incontinence have abnormal resting tone of the internal anal sphincter that is responsible for maintaining continence. Sensation of rectal distension also appears to be diminished in patients with diabetes, impairing the recto-anal reflex that leads to relaxation of the internal sphincter. ${ }^{70}$ Furthermore, studies show that hyperglycemia independently impairs internal anal sphincter tone and rectal compliance, potentiating underlying deficits. ${ }^{71}$ 


\section{Evaluation of Fecal Incontinence}

Evaluation for fecal incontinence should include an evaluation for alternative etiologies for incontinence, including fecal impaction, infection, colonic mucosal disease, or primary diarrhea. A rectal examination will indicate whether fecal impaction with overflow incontinence is responsible for the symptoms and will allow for assessment of the anal sphincter. Evaluation of diarrhea should include stool cultures and flexible sigmoidoscopy or colonoscopy to evaluate for mucosal inflammation or structural etiology for the incontinence. $^{72}$

\section{Diagnosis of Fecal Incontinence}

Anorectal manometry is the primary method used to define the deficits in fecal incontinence and typically quantifies sphincter pressure, rectal sensation, rectal compliance, and rectal reflexes. Recent technical advances in high-resolution manometry have greatly enhanced the ability to define underlying mechanisms causing fecal incontinence and may guide potential biofeedback therapy. ${ }^{72}$

Management of fecal incontinence primarily begins with optimization of glycemic control and initiation of antidiarrheal medications. Loperamide and diphenoxylate with atropine reduce incontinence episodes and may suffice in managing modest symptoms.

Cholestyramine may decrease diarrhea and improve continence. Biofeedback therapy aims to train individuals to become more aware of, and increase control of, anorectal muscles. It may improve anal sphincter strength, coordination of muscles, and sensory perception. Clinical improvement appears to be variable, as a result of differing symptom severity and lack of uniformity in biofeedback therapy. Small non-randomized studies of biofeedback have shown clinical efficacy in diabetic patients with fecal incontinence. ${ }^{73}$ In refractory fecal incontinence, sacral nerve stimulation device implantation and surgery may improve symptoms; however, studies of fecal incontinence in diabetic patients have not been performed.

\section{HEPATOBILIARY DISORDERS}

\section{Nonalcoholic Fatty Liver Disease Overview}

Nonalcoholic fatty liver disease (NAFLD) is defined by fat deposition in the liver in the absence of alcohol abuse or other known hepatotoxins. It is a spectrum that includes nonalcoholic fatty liver (NAFL; fat deposition in the liver without inflammation) and nonalcoholic steatohepatitis (NASH; fat deposition with associated inflammation or hepatitis). Fatty liver is typically defined by the accumulation of fat in the liver without associated inflammation or elevated liver enzymes. In contrast, NASH is defined by the presence of fatty infiltrate in the liver with associated inflammation. Distinguishing between these 2 entities remains a significant clinical challenge (Table 3).

\section{Epidemiology}

Incidence of NAFLD in the general population is not well defined. The incidence reported by different studies varies dramatically depending on the population, definition of NAFLD, and diagnostic modalities. Based on 2 histology-based studies of potential living liver 
donors, the prevalence of NAFLD is estimated to be between $20 \%$ and $51 \% .74,75$ The prevalence of NAFLD in DM2 may be as high as $69 \%$, with diabetes as an independent predictor for progression of liver disease. ${ }^{76}$ Risk factors include obesity, diabetes, hyperlipidemia, and age. The highest incidence appears to be in Hispanic individuals, and the lowest incidence occurs in African American individuals. ${ }^{76}$

\section{Natural History of NAFLD}

Based on limited studies, individuals with NAFLD have increased mortality as compared with matched control populations. Although liver-associated mortality is higher in NAFLD, the most common cause of death in this population is cardiovascular disease. ${ }^{77}$ The survival of patients with NASH with associated inflammation as compared with those with benign hepatic fat is significantly worse. ${ }^{78}$ Individuals with NASH, but probably not NAFL, have a significant risk of progression to cirrhosis, which will occur in up to $30 \% .{ }^{23}$

\section{Pathogenesis of NAFLD}

The pathogenesis of NAFLD is poorly understood, and the lack of adequate animal models has limited research. The accumulation of fat in the liver appears to be related to an imbalance where lipogenesis exceeds lipolysis, and the amount of hepatic free-fatty acids exceeds requirements for mitochondrial oxidation, cholesterol synthesis, and phospholipid synthesis. This process appears to be driven by insulin levels and insulin resistance that drives free-fatty acid synthesis, inhibits synthesis of very low-density lipoproteins (VLDL), and enhances susceptibility to hepatic injury. ${ }^{79}$ Free-fatty acids are toxic to hepatocytes through multiple mechanisms and play a role in induction of inflammation, but the precise mechanism underlying the progression from fatty liver to steatohepatitis is not completely understood, although it may be related to oxidative stress and mitochondrial dysfunction. 66,80

\section{Signs and Symptoms of NAFLD}

NAFLD is typically asymptomatic and is frequently incidentally detected during an evaluation for an unrelated condition. Some individuals may report right upper quadrant abdominal pain, fatigue, or malaise. Although patients may have an enlarged liver on physical examination, concomitant obesity makes this physical finding difficult to appreciate. Serum alanine aminotransferase (ALT) and aspartate aminotransferase (AST) are frequently elevated, with ALT usually higher than AST. In the absence of advanced liver disease, total bilirubin, prothrombin time, and albumin levels remain normal. Imaging studies may show fat infiltrating the liver with the appearance varying by imaging modality.

Diagnosis of NAFLD or NASH is a clinical and pathologic diagnosis. Clinically, practitioners must exclude other etiologies for liver disease, including significant alcohol use, medications causing hepatic injury, viral hepatitis, Wilson's disease, autoimmune hepatitis, hemochromatosis, or alternative etiologies. The definition of significant alcohol use varies by gender and ultimately becomes a clinical assessment. Without histology, one cannot distinguish between benign fatty liver deposition and NASH, the latter being associated with a worse prognosis. 


\section{New Diagnostic Tools for NAFLD}

Although practitioners typically begin with abdominal ultrasound when evaluating those suspected for NAFLD, this modality is not sensitive, nor specific. Computed to-mography and magnetic resonance imaging (MRI) are more sensitive and specific in detecting steatosis. Newer forms of MRI are being developed to detect individuals with NASH or advanced fibrosis. Specifically, MR transient elastography measures liver stiffness noninvasively and successfully detects the presence of fibrosis in NAFLD, although is less accurate in the setting of obesity. ${ }^{24}$ Other composite scores of simple laboratory parameters are being validated for detecting advanced fibrosis. ${ }^{69}$

\section{The Role of Liver Biopsy}

The role of liver biopsy in diagnosis remains somewhat debated. In some cases, biopsy is clearly needed to rule out concomitant liver disease. Although liver biopsy is an expensive and morbid procedure, it remains the gold standard for diagnosis and staging of disease. Fat deposition in the form of steatosis may be present on histology in fatty liver and NASH, but cellular damage with hepatocyte ballooning and/or fibrosis occurs only in NASH and distinguishes the progressive disease from benign fat infiltration. An active area of investigation is the development of noninvasive testing to identify NASH or advanced fibrosis. ${ }^{81}$

\section{Management of NAFLD}

Current management involves addressing any modifiable risk factors for NAFLD, including insulin resistance, diabetes, obesity, and hyperlipidemia, as well as the liver disease.

Medications aimed at reversing the liver disease are reserved for individuals with NASH.

\section{Lifestyle Modification}

First-line treatment for NAFLD is initiation of diet and exercise. Based on current studies, weight loss of $3 \%$ to $5 \%$ of body weight may improve steatosis, but greater weight loss may be required to improve fibrosis. Exercise even without weight loss improves steatosis but may not reverse fibrosis. ${ }^{77}$

\section{Medical Therapies for NASH and Diabetes}

The medical therapies for NASH evaluated to date focus on enhancing insulin sensitivity but have had limited success. Metformin initially showed promise; however, a meta-analysis shows that metformin does not decrease liver enzymes or improve histology. ${ }^{76}$ Conversely, a meta-analysis of 4 randomized clinical trials concluded that pioglitazone, a thiazolidinedione (TZD), significantly reduced steatosis and inflammation in NASH. However, the trials enrolled patients without diabetes, limiting the generalizability to diabetic patients, and longterm cardiovascular safety data are lacking. ${ }^{77}$ Furthermore, recent associations of TZDs with bladder cancer have limited their overall use. ${ }^{82}$ Similarly, vitamin E (800 IU per day) may improve histology in NASH; however, few data support use of vitamin $\mathrm{E}$ in diabetic patients with NASH, particularly given the potential association with prostate cancer. ${ }^{83}$ Bariatric surgery appears to improve liver histology and will likely be a future treatment; however, randomized clinical trials are needed to support its use and determine which surgeries are 
most efficacious. ${ }^{84}$ Overall, studies of NASH in diabetic patients are lacking and will be essential going forward to guide management; however, there are no approved treatments currently for liver disease associated with NASH.

\section{Statin Use in NAFLD}

Central to management of NAFLD is risk factor modification. Statins are extremely effective for dyslipidemia but may cause elevations in AST and ALT. As a result of this potential side effect, practitioners may be reluctant to use statins in the setting of NAFLD. The use of statins in the setting of NAFLD appears safe without increased risk of hepatotoxicity, and post hoc analyses suggest that statins improve cardiovascular outcomes and liver enzymes in NAFLD. ${ }^{77}$ Although there are no guidelines to guide practitioners, a twofold increase in liver enzymes should be tolerated without cessation of therapy.

\section{Cholelithiasis and Association with Diabetes}

Gallstone disease and its complications, such as cholangitis, cholecystitis, and gallstone pancreatitis, occur more frequently in diabetic patients based on epidemiologic studies. However, these 2 diseases are both quite common and share risk factors, making it difficult to tease out the relationship between these entities. Mouse and human studies have shown relative bile stasis within the gallbladder and reduced cholecystokinin levels that may drive gallbladder stone formation. ${ }^{85}$ Prophylactic cholecystectomy should not be performed for asymptomatic gallstones in diabetic patients.

\section{PANCREATIC DISEASE}

\section{Pancreatic Insufficiency}

Pancreatic exocrine insufficiency occurs in patients with DM1 and DM2. Historical studies demonstrated biochemical insufficiency in nearly $50 \%$ and $30 \%$ to $50 \%$ of patients with insulin-dependent diabetes and noninsulin-dependent diabetes, respectively. ${ }^{86}$ Most of these patients have minor pancreatic exocrine insufficiency that is not clinically significant. If exocrine insufficiency is clinically significant and leads to fat malabsorption, individuals may benefit from mealtime pancreatic enzyme supplementation containing amylase, lipase, and protease. Clinically, pancreatic insufficiency may be a difficult entity to diagnose, and at times a clinical trial of pancreatic enzyme supplementation may be appropriate.

\section{Acute Pancreatitis in Diabetes}

Diabetic patients have an elevated risk of pancreatitis that appears to be intrinsic to the diseases as well as related to medications. Patients with DM1 have a twofold increased risk of pancreatitis, and patients with DM2 have a nearly threefold increased risk of pancreatitis as compared with the general population. The highest risk was in patients with DM2 who were younger than 30 years old. ${ }^{66,87}$ The precise mechanism is not completely understood but may be related to ongoing inflammation. DM2 and pancreatitis share obesity and hypertriglyceridemia as common risk factors. Based on a review of the FDA adverse event reporting, GLP-1 agonists and dipeptidyl peptidase 4 inhibitors are associated with a sixfold increased risk of pancreatitis with a possible increased risk of pancreatic cancer. ${ }^{88}$ 


\section{Pancreatic Cancer}

Diabetes is a risk factor for pancreatic cancer but may also be a presenting sign. The etiologic role of diabetes in development of pancreatic cancer is poorly understood, although may be related to low-grade chronic inflammation of the pancreas. The relative risk of pancreatic cancer appears to be nearly 2 in patients with diabetes. The highest risk was in newly diagnosed diabetic patients, underscoring that new-onset diabetes may be an early sign of pancreatic malignancy. ${ }^{89}$

\section{SUMMARY}

The gastrointestinal complications of diabetes can affect essentially any organ in the gastrointestinal tract, are associated with significant morbidity and mortality, and can directly impair quality of life. With this in mind, the health care professional should be aware of these associated conditions, as well as the high prevalence of overlapping gastrointestinal symptoms unrelated to diabetes. Although screening for heart disease, retinopathy, neuropathy, and nephropathy typically make their way into pre-formed electronic records for a clinic visit, gastrointestinal symptoms are frequently not screened for and may be underreported. Abnormalities uncovered should be investigated with consideration of referral to a gastroenterology specialist when appropriate. In this way, screening and treating gastrointestinal-related complications may enhance comprehensive care of diabetic patients.

\section{Funding Sources:}

None.

\section{REFERENCES}

1. Camilleri M, Dubois D, Coulie B, et al. Prevalence and socioeconomic impact of upper gastrointestinal disorders in the United States: results of the US Upper Gastrointestinal Study. Clin Gastroenterol Hepatol 2005;3:543-52. [PubMed: 15952096]

2. Gatopoulou A, Papanas N, Maltezos E. Diabetic gastrointestinal autonomic neuropathy: current status and new achievements for everyday clinical practice. Eur J Intern Med 2012;23:499-505. [PubMed: 22863425]

3. Kinekawa F, Kubo F, Matsuda K, et al. Relationship between esophageal dysfunction and neuropathy in diabetic patients. Am J Gastroenterol 2001;96: 2026-32. [PubMed: 11467628]

4. Lluch I, Ascaso JF, Mora F, et al. Gastroesophageal reflux in diabetes mellitus. Am J Gastroenterol 1999;94:919-24. [PubMed: 10201457]

5. Wang X, Pitchumoni CS, Chandrarana K, et al. Increased prevalence of symptoms of gastroesophageal reflux diseases in type 2 diabetics with neuropathy. World J Gastroenterol 2008;14:709-12. [PubMed: 18205259]

6. Singh PK, Hota D, Dutta P, et al. Pantoprazole improves glycemic control in type 2 diabetes: a randomized, double-blind, placebo-controlled trial. J Clin Endocrinol Metab 2012;97:E2105-8. [PubMed: 22904177]

7. Wilcox CM, Karowe MW. Esophageal infections: etiology, diagnosis, and management. Gastroenterologist 1994;2:188-206. [PubMed: 7987618]

8. Jung HK, Choung RS, Locke GR 3rd, et al. The incidence, prevalence, and outcomes of patients with gastroparesis in Olmsted County, Minnesota, from 1996 to 2006. Gastroenterology 2009;136:1225-33. [PubMed: 19249393] 
9. Choung RS, Locke GR 3rd, Schleck CD, et al. Risk of gastroparesis in subjects with type 1 and 2 diabetes in the general population. Am J Gastroenterol 2012; 107:82-8. [PubMed: 22085818]

10. Keshavarzian A, Iber FL, Vaeth J. Gastric emptying in patients with insulin-requiring diabetes mellitus. Am J Gastroenterol 1987;82:29-35. [PubMed: 3799578]

11. Camilleri M, Bharucha AE, Farrugia G. Epidemiology, mechanisms, and management of diabetic gastroparesis. Clin Gastroenterol Hepatol 2011;9:5-12 [quiz: e17]. [PubMed: 20951838]

12. Schvarcz E, Palmer M, Aman J, et al. Physiological hyperglycemia slows gastric emptying in normal subjects and patients with insulin-dependent diabetes mellitus. Gastroenterology 1997;113:60-6. [PubMed: 9207262]

13. Farrugia G Interstitial cells of Cajal in health and disease. Neurogastroenterol Motil 2008;20(Suppl 1):54-63. [PubMed: 18402642]

14. Harberson J, Thomas RM, Harbison SP, et al. Gastric neuromuscular pathology in gastroparesis: analysis of full-thickness antral biopsies. Dig Dis Sci 2010;55: 359-70. [PubMed: 19997975]

15. Schwartz TW. Pancreatic polypeptide: a hormone under vagal control. Gastroenterology 1983;85:1411-25. [PubMed: 6138294]

16. Tomita R, Tanjoh K, Fujisaki S, et al. The role of nitric oxide (NO) in the human pyloric sphincter. Hepatogastroenterology 1999;46:2999-3003. [PubMed: 10576391]

17. Watkins CC, Sawa A, Jaffrey S, et al. Insulin restores neuronal nitric oxide synthase expression and function that is lost in diabetic gastropathy. J Clin Invest 2000;106:803. [PubMed: 10995791]

18. Choi KM, Gibbons SJ, Nguyen TV, et al. Heme oxygenase-1 protects interstitial cells of Cajal from oxidative stress and reverses diabetic gastroparesis. Gastroenterology 2008;135:2055-64, 2064.e1-2. [PubMed: 18926825]

19. Sarnelli G, Caenepeel P, Geypens B, et al. Symptoms associated with impaired gastric emptying of solids and liquids in functional dyspepsia. Am J Gastroenterol 2003;98:783-8. [PubMed: 12738456]

20. Camilleri M, Parkman HP, Shafi MA, et al., American College of Gastroenterology. Clinical guideline: management of gastroparesis. Am J Gastroenterol 2013;108: 18-37 [quiz: 38]. [PubMed: 23147521]

21. Abell TL, Camilleri M, Donohoe K, et al., American Neurogastroenterology and Motility Society and the Society of Nuclear Medicine. Consensus recommendations for gastric emptying scintigraphy: a joint report of the American Neurogastroenterology and Motility Society and the Society of Nuclear Medicine. J Nucl Med Technol 2008;36:44-54. [PubMed: 18287197]

22. Fraser RJ, Horowitz M, Maddox AF, et al. Hyperglycaemia slows gastric emptying in type 1 (insulin-dependent) diabetes mellitus. Diabetologia 1990; 33:675-80. [PubMed: 2076799]

23. Fassio E, Alvarez E, Dominguez N, et al. Natural history of nonalcoholic steatohepatitis: a longitudinal study of repeat liver biopsies. Hepatology 2004;40: 820-6. [PubMed: 15382171]

24. Musso G, Gambino R, Cassader M, et al. Meta-analysis: natural history of nonalcoholic fatty liver disease (NAFLD) and diagnostic accuracy of non-invasive tests for liver disease severity. Ann Med 2011;43:617-49. [PubMed: 21039302]

25. Lee A, Kuo B. Metoclopramide in the treatment of diabetic gastroparesis. Expert Rev Endocrinol Metab 2010;5:653-62. [PubMed: 21278804]

26. McCallum RW, Ricci DA, Rakatansky H, et al. A multicenter placebo-controlled clinical trial of oral metoclopramide in diabetic gastroparesis. Diabetes Care 1983;6:463-7. [PubMed: 6400707]

27. Perkel MS, Moore C, Hersh T, et al. Metoclopramide therapy in patients with delayed gastric emptying: a randomized, double-blind study. Dig Dis Sci 1979;24: 66 2-6.

28. Snape WJ Jr, Battle WM, Schwartz SS, et al. Metoclopramide to treat gastroparesis due to diabetes mellitus: a double-blind, controlled trial. Ann Intern Med 1982;96:444-6. [PubMed: 7065559]

29. Miller LG, Jankovic J. Metoclopramide-induced movement disorders. Clinical findings with a review of the literature. Arch Intern Med 1989;149:2486-92. [PubMed: 2684075]

30. Bateman DN, Rawlins MD, Simpson JM. Extrapyramidal reactions with metoclopramide. Br Med J (Clin Res Ed) 1985;291:930-2. 
31. Parkman HP, Mishra A, Jacobs M, et al. Clinical response and side effects of metoclopramide: associations with clinical, demographic, and pharmacogenetic parameters. J Clin Gastroenterol 2012;46:494-503. [PubMed: 22688145]

32. Silvers D, Kipnes M, Broadstone V, et al. Domperidone in the management of symptoms of diabetic gastroparesis: efficacy, tolerability, and quality-of-life outcomes in a multicenter controlled trial. DOM-USA-5 Study Group. Clin Ther 1998;20:438-53.

33. Patterson D, Abell T, Rothstein R, et al. A double-blind multicenter comparison of domperidone and metoclopramide in the treatment of diabetic patients with symptoms of gastroparesis. Am J Gastroenterol 1999;94:1230-4. [PubMed: 10235199]

34. Franzese A, Borrelli O, Corrado G, et al. Domperidone is more effective than cisapride in children with diabetic gastroparesis. Aliment Pharmacol Ther 2002;16: 951- 7. [PubMed: 11966504]

35. Janssens J, Peeters TL, Vantrappen G, et al. Improvement of gastric emptying in diabetic gastroparesis by erythromycin. Preliminary studies. N Engl J Med 1990;322:1028-31. [PubMed: 2320062]

36. Adams LA, Angulo P. Role of liver biopsy and serum markers of liver fibrosis in non-alcoholic fatty liver disease. Clin Liver Dis 2007;11:25-35, viii. [PubMed: 17544970]

37. Abell T, McCallum R, Hocking M, et al. Gastric electrical stimulation for medically refractory gastroparesis. Gastroenterology 2003;125:421-8. [PubMed: 12891544]

38. Chu H, Lin Z, Zhong L, et al. Treatment of high-frequency gastric electrical stimulation for gastroparesis. J Gastroenterol Hepatol 2012;27:1017-26. [PubMed: 22128901]

39. Wang CP, Kao CH, Chen WK, et al. A single-blinded, randomized pilot study evaluating effects of electroacupuncture in diabetic patients with symptoms suggestive of gastroparesis. J Altern Complement Med 2008;14:833-9. [PubMed: 18721079]

40. Sjoberg K, Eriksson KF, Bredberg A, et al. Screening for coeliac disease in adult insulin-dependent diabetes mellitus. J Intern Med 1998;243:133-40. [PubMed: 9566642]

41. Talal AH, Murray JA, Goeken JA, et al. Celiac disease in an adult population with insulindependent diabetes mellitus: use of endomysial antibody testing. Am J Gastroenterol 1997;92:1280-4. [PubMed: 9260789]

42. Cronin CC, Feighery A, Ferriss JB, et al. High prevalence of celiac disease among patients with insulin-dependent (type I) diabetes mellitus. Am J Gastroenterol 1997;92:2210-2. [PubMed: 9399754]

43. Fasano A, Berti I, Gerarduzzi T, et al. Prevalence of celiac disease in at-risk and not-at-risk groups in the United States: a large multicenter study. Arch Intern Med 2003;163:286-92. [PubMed: 12578508]

44. Ludvigsson JF, Rubio-Tapia A, van Dyke CT, et al. Increasing incidence of celiac disease in a North American population. Am J Gastroenterol 2013;108(5):818-24. [PubMed: 23511460]

45. Cronin CC, Shanahan F. Insulin-dependent diabetes mellitus and coeliac disease. Lancet 1997;349:1096-7. [PubMed: 9107261]

46. Green PH, Cellier C. Celiac disease. N Engl J Med 2007;357:1731-43. [PubMed: 17960014]

47. Kagnoff MF. Celiac disease: pathogenesis of a model immunogenetic disease. J Clin Invest 2007;117:41-9. [PubMed: 17200705]

48. Rostom A, Dube C, Cranney A, et al. Celiac disease. Evid Rep Technol Assess (Summ) 2004; (104):1-6.

49. Sud S, Marcon M, Assor E, et al. 2010 Celiac disease and pediatric type 1 diabetes: diagnostic and treatment dilemmas. Int J Pediatr Endocrinol 2010;2010: 161285. [PubMed: 20652072]

50. Nakajima K 2012 Recent advances in dermatitis herpetiformis. Clin Dev Immunol 2012;2012:914162. [PubMed: 22811741]

51. van der Windt DA, Jellema P, Mulder CJ, et al. Diagnostic testing for celiac disease among patients with abdominal symptoms: a systematic review. JAMA 2010;303:1738-46. [PubMed: 20442390]

52. Rostom A, Murray JA, Kagnoff MF. American Gastroenterological Association (AGA) Institute technical review on the diagnosis and management of celiac disease. Gastroenterology 2006; 131:1981-2002. [PubMed: 17087937] 
53. Rostom A, Dube C, Cranney A, et al. The diagnostic accuracy of serologic tests for celiac disease: a systematic review. Gastroenterology 2005;128:S38-46. [PubMed: 15825125]

54. Corrao G, Corazza GR, Bagnardi V, et al. Mortality in patients with coeliac disease and their relatives: a cohort study. Lancet 2001;358:356-61. [PubMed: 11502314]

55. Loftus CG, Loftus EV Jr. Cancer risk in celiac disease. Gastroenterology 2002; 123:1726-9. [PubMed: 12404246]

56. Leffler DA, Dennis M, Hyett B, et al. Etiologies and predictors of diagnosis in nonresponsive celiac disease. Clin Gastroenterol Hepatol 2007;5:445-50. [PubMed: 17382600]

57. Parlesak A, Klein B, Schecher K, et al. Prevalence of small bowel bacterial over-growth and its association with nutrition intake in nonhospitalized older adults. J Am Geriatr Soc 2003;51:768 73. [PubMed: 12757562]

58. Virally-Monod M, Tielmans D, Kevorkian JP, et al. Chronic diarrhoea and diabetes mellitus: prevalence of small intestinal bacterial overgrowth. Diabete Metab 1998;24:530-6. [PubMed: 9932220]

59. Dukowicz AC, Lacy BE, Levine GM. Small intestinal bacterial overgrowth: a comprehensive review. Gastroenterol Hepatol 2007;3:112-22.

60. Saltzman JR, Russell RM. Nutritional consequences of intestinal bacterial over-growth. Compr Ther 1994;20:523-30. [PubMed: 7805370]

61. Bures J, Cyrany J, Kohoutova D, et al. Small intestinal bacterial overgrowth syndrome. World J Gastroenterol 2010;16:2978-90. [PubMed: 20572300]

62. Corazza GR, Menozzi MG, Strocchi A, et al. The diagnosis of small bowel bacterial overgrowth. Reliability of jejunal culture and inadequacy of breath hydrogen testing. Gastroenterology 1990;98:302-9. [PubMed: 2295385]

63. Pimentel M Review of rifaximin as treatment for SIBO and IBS. Expert Opin Investig Drugs 2009;18:349-58.

64. Lysy J, Israeli E, Goldin E. The prevalence of chronic diarrhea among diabetic patients. Am J Gastroenterol 1999;94:2165-70. [PubMed: 10445544]

65. Miller LJ. Small intestinal manifestations of diabetes mellitus. Yale J Biol Med 1983;56:189-93. [PubMed: 6659561]

66. Feldman M, Friedman LS, Brandt LJ, editors. Sleisenger and Fordtran's gastrointesitnal and liver disease: pathophysiology/diagnosis/management. 9th edition. Philadelphia: Elsevier; 2010.

67. Fedorak RN, Field M, Chang EB. Treatment of diabetic diarrhea with clonidine. Ann Intern Med 1985;102:197-9. [PubMed: 3966758]

68. Meyer C, O’Neal DN, Connell W, et al. Octreotide treatment of severe diabetic diarrhoea. Intern Med J 2003;33:617-8. [PubMed: 14656240]

69. Angulo P, Hui JM, Marchesini G, et al. The NAFLD fibrosis score: a noninvasive system that identifies liver fibrosis in patients with NAFLD. Hepatology 2007;45: 846-54. [PubMed: 17393509]

70. Schiller LR, Santa Ana CA, Schmulen AC, et al. Pathogenesis of fecal incontinence in diabetes mellitus: evidence for internal-anal-sphincter dysfunction. N Engl J Med 1982;307:1666-71. [PubMed: 7144865]

71. Russo A, Botten R, Kong MF, et al. Effects of acute hyperglycaemia on anorectal motor and sensory function in diabetes mellitus. Diabet Med 2004;21:176-82. [PubMed: 14984454]

72. Rao SS. Diagnosis and management of fecal incontinence. American College of Gastroenterology Practice Parameters Committee. Am J Gastroenterol 2004;99: 1585-604. [PubMed: 15307881]

73. Wald A, Tunuguntla AK. Anorectal sensorimotor dysfunction in fecal incontinence and diabetes mellitus. Modification with biofeedback therapy. N Engl J Med 1984;310:1282-7. [PubMed: 6717494]

74. Lee JY, Kim KM, Lee SG, et al. Prevalence and risk factors of non-alcoholic fatty liver disease in potential living liver donors in Korea: a review of 589 consecutive liver biopsies in a single center. J Hepatol 2007;47:239-44. [PubMed: 17400323]

75. Marcos A, Fisher RA, Ham JM, et al. Selection and outcome of living donors for adult to adult right lobe transplantation. Transplantation 2000;69:2410-5. [PubMed: 10868650] 
76. Vernon G, Baranova A, Younossi ZM. Systematic review: the epidemiology and natural history of non-alcoholic fatty liver disease and non-alcoholic steatohepatitis in adults. Aliment Pharmacol Ther 2011;34:274-85. [PubMed: 21623852]

77. Chalasani N, Younossi Z, Lavine JE, et al., American Gastroenterological Association, American Association for the Study of Liver Diseases, American College of Gastroenterologyh. The diagnosis and management of non-alcoholic fatty liver disease: practice guideline by the American Gastroenterological Association, American Association for the Study of Liver Diseases, and American College of Gastroenterology. Gastroenterology 2012;142:1592-609. [PubMed: 22656328]

78. Soderberg C, Stal P, Askling J. Decreased survival of subjects with elevated liver function tests during a 28-year follow-up. Hepatology 2010;51:595-602. [PubMed: 20014114]

79. Chitturi S, Abeygunasekera S, Farrell GC, et al. NASH and insulin resistance: insulin hypersecretion and specific association with the insulin resistance syndrome. Hepatology 2002;35:373-9. [PubMed: 11826411]

80. Browning JD, Horton JD. Molecular mediators of hepatic steatosis and liver injury. J Clin Invest 2004;114:147-52. [PubMed: 15254578]

81. Farrell GC, Larter CZ. Nonalcoholic fatty liver disease: from steatosis to cirrhosis. Hepatology 2006;43:S99-112. [PubMed: 16447287]

82. Lewis JD, Ferrara A, Peng T, et al. Risk of bladder cancer among diabetic patients treated with pioglitazone: interim report of a longitudinal cohort study. Diabetes Care 2011;34:916-22. [PubMed: 21447663]

83. Sanyal AJ, Chalasani N, Kowdley KV, et al. Pioglitazone, vitamin E, or placebo for nonalcoholic steatohepatitis. N Engl J Med 2010;362:1675-85. [PubMed: 20427778]

84. Chavez-Tapia NC, Tellez-Avila FI, Barrientos-Gutierrez T, et al. Bariatric surgery for non-alcoholic steatohepatitis in obese patients. Cochrane Database Syst Rev 2010;(1):CD007340. [PubMed: 20091629]

85. Pazzi P, Scagliarini R, Gamberini S, et al. Review article: gall-bladder motor function in diabetes mellitus. Aliment Pharmacol Ther 2000;14(Suppl 2):62-5. [PubMed: 10903007]

86. Hardt PD, Ewald N. 2011 Exocrine pancreatic insufficiency in diabetes mellitus: a complication of diabetic neuropathy or a different type of diabetes? Exp Diabetes Res 2011;2011:761950. [PubMed: 21822421]

87. Shen HN, Chang YH, Chen HF, et al. Increased risk of severe acute pancreatitis in patients with diabetes. Diabet Med 2012;29:1419-24. [PubMed: 22506974]

88. Elashoff M, Matveyenko AV, Gier B, et al. Pancreatitis, pancreatic, and thyroid cancer with glucagon-like peptide-1-based therapies. Gastroenterology 2011; 141:150-6. [PubMed: 21334333]

89. Ben Q, Xu M, Ning X, et al. Diabetes mellitus and risk of pancreatic cancer: a meta-analysis of cohort studies. Eur J Cancer 2011;47:1928-37. [PubMed: 21458985] 


\section{KEY POINTS}

- Gastrointestinal symptoms are common in the general population and frequently are related to irritable bowel syndrome.

- The incidence of multiple gastrointestinal diseases is more common in diabetic patients for a variety of reasons.

- Diabetic neuropathy may lead to abnormal gastrointestinal motility that causes gastroparesis, small bacterial intestinal overgrowth, diabetic diarrhea, and fecal incontinence.

\section{Risk Factors for Gastroesophageal Reflux in Diabetes}

- Cardiovascular autonomic dysfunction

- $\quad$ Elevated body mass index

- $\quad$ Longer duration of disease

- $\quad$ Poor glycemic control

\section{Signs and Symptoms of Candidiasis}

- $\quad$ Odynophagia

- Dysphagia with solids

- $\quad$ Avoidance of oral intake

- Erythematous friable lesions in oropharynx

- White patches in oropharynx

\section{Symptoms of Gastroparesis}

- $\quad$ Nausea

- $\quad$ Vomiting

- $\quad$ Early satiety

- $\quad$ Bloating

- $\quad$ Upper abdominal discomfort

\section{Diagnosis of Gastroparesis}

- Symptoms of gastroparesis

- Absence of gastric outlet obstruction based on imaging or endoscopy

- Presence of retained food in the stomach on endoscopy after a fast of more than 12 hours

- $\quad$ Slowed gastric emptying based on scintigraphy

\section{Management of Gastroparesis}


- $\quad$ Evaluation and correction of nutritional status

- Improvement of gastric emptying

- $\quad$ Symptomatic treatment

\section{Adult Presentation of Celiac Disease}

- Diarrhea

- $\quad$ Steatorrhea

- Abdominal pain

- Weight loss

\section{Symptoms of Diabetic Diarrhea}

- Watery, voluminous diarrhea

- $\quad$ Nocturnal symptoms

- $\quad$ Steatorrhea

- $\quad$ Fecal incontinence

- Episodic symptom s with intervening normal bowel habits or constipation

- Absence of abdominal pain

\section{Treatment of Fecal Incontinence}

- Optimization of glycemic control

- Supportive lifestyle modification

- $\quad$ Antidiarrheal medications

- $\quad$ Biofeedback therapy

- Sacral stimulator or surgery

\section{Definition of NAFLD}

- Presence of hepatic steatosis based on imaging or histology

- Absence of significant alcohol use

- Absence of secondary etiologies for liver disease

- Absence of coexisting chronic liver disease 


\section{Table 1}

Summary of prokinetic medications for gastroparesis

\begin{tabular}{llll}
\hline Drug & Starting Dose & Side Effects & Considerations \\
\hline Metoclopramide & $5 \mathrm{mg}$ with meals & $\begin{array}{l}\text { Tardive dyskinesia, QT prolongation, akathisia, } \\
\text { parkinsonian movements, hyperprolactinemia }\end{array}$ & $\begin{array}{l}\text { Use minimum effective dose }>4 \text { wk of use needs } \\
\text { to be re-evaluated }\end{array}$ \\
\hline Domperidone & $10 \mathrm{mg}$ with meals & QT prolongation, hyperprolactinemia & $\begin{array}{l}\text { Baseline electrocardiogram Not readily available } \\
\text { in the United States Better side-effect profile }\end{array}$ \\
\hline Erythromycin & $3 \mathrm{mg} / \mathrm{kg}$ intravenous & Tachyphylaxis & Evidence supports short-term use in hospital \\
\hline
\end{tabular}




\section{Table 2}

Celiac disease

\begin{tabular}{ll}
\hline $\begin{array}{l}\text { Type of Celiac } \\
\text { Disease }\end{array}$ & Definition \\
\hline Classic & Patients with abnormal duodenal histology with typical gastrointestinal symptoms \\
\hline Atypical & $\begin{array}{l}\text { Lack of gastrointestinal symptoms but may present with extraintestinal findings related to celiac disease (eg, iron } \\
\text { deficiency anemia, dermatitis herpetiformis) }\end{array}$ \\
\hline Silent & Diagnosed based on serologies or during endoscopy for another indication. Lack of gastrointestinal symptoms \\
\hline Latent & $\begin{array}{l}\text { Individual with celiac disease on gluten-free diet with resolution of symptoms and duodenal changes based on histology } \\
\text { or patients with normal duodenal histology with gluten-containing diet who will later develop celiac disease }\end{array}$ \\
\hline Potential & $\begin{array}{l}\text { Individuals with positive serologies but negative duodenal biopsies without symptoms who are at high risk for } \\
\text { development of classic celiac disease }\end{array}$ \\
\hline
\end{tabular}




\section{Table 3}

Spectrum of nonalcoholic fatty liver disease

\begin{tabular}{ll}
\hline Term & Definition \\
\hline Nonalcoholic fatty liver disease (NAFLD) & Encompasses spectrum of liver disease from fatty infiltrate without inflammation to cirrhosis \\
\hline Nonalcoholic fatty liver (NAFL) & Liver fat accumulation in the liver without inflammation \\
\hline Nonalcoholic steatohepatitis (NASH) & Liver fat accumulation with inflammation \\
\hline NASH cirrhosis & End stage of NASH \\
\hline
\end{tabular}

Nonalcoholic fatty liver disease (NAFLD)

Encompasses spectrum of liver disease from fatty infiltrate without inflammation to cirrhosis

Liver fat accumulation with inflammation 